Research Article

\title{
Family violence as a determinant of suicidality and depression among adolescents in India
}

\author{
Nandita Bhan, Rupa Jose, Jay G Silverman, Anita Raj \\ Keywords: global health \\ https://doi.org/10.29392/joghr.3.e2019072
}

\section{Journal of Global Health Reports}

Vol. 3, 2019

\begin{abstract}
Background
Suicide/self-harm is a leading cause of death for adolescent girls and boys globally, and family violence may increase the risk for adolescent suicidality, self-harm and depression. This study assessed whether family violence was associated with suicidality and depressive symptoms among adolescents in India. This study is the first to examine the role of family violence in suicidality and depression among Indian adolescents.
\end{abstract}

\section{Methods}

We analyzed data from 20,594 adolescents (10-19 years) collected as part of a cross-sectional study in Bihar and Uttar Pradesh, India (2015-2016). Family violence variables included child abuse, witnessing parental marital violence, and personal experiences of marital violence. Suicidal ideation, self-harm and depression (measured using the Patient Health Questionnaire (PHQ-9)) served as outcomes of interest. Multivariate models assessed associations of family violence with outcomes, adjusting for sociodemographic determinants. Analyses were stratified by adolescent gender, age (10-14 and 15-19 year olds) and marital status (for girls 15-19 years).

\begin{abstract}
Results
Married adolescent girls in India were at highest risk, with 8.2\% reporting suicidal ideation and $4.9 \%$ reporting self-harm behaviors. Unmarried older adolescent girls were more likely to consider suicide compared to same-age boys (4.4\% vs $2.4 \%$ respectively) while older boys (4.2\% vs $2.9 \%$ for older girls) and younger adolescents (boys:5.3\%, girls: $6.1 \%$ ) were more likely to engage in self-harm behaviors. Depressive symptoms were rare except among older married (8\%) and unmarried (5\%) girls. For unmarried girls, child abuse was associated with suicidality (adjusted odds ratio $(\mathrm{AOR})=2.08,95 \%$ confidence interval $\mathrm{CI}=1.4-3.07$ ) and self-harm behaviors ( $\mathrm{AOR}=2.8,95 \% \mathrm{CI}=1.83-4.26$ ) and witnessing parental marital violence was associated with moderate-severe depressive symptoms $(\mathrm{AOR}=1.81,95 \% \mathrm{CI}=1.30-2.50)$. For married girls aged $15-19$ years, personal experiences of marital violence were associated with suicidality (AOR=3.73, 95\% $\mathrm{CI}=2.0-6.96)$ and moderate-severe depressive symptoms ( $\mathrm{AOR}=2.93,95 \% \mathrm{CI}=1.83-4.68$ ). Among unmarried boys 13-19 years, witnessing parental marital violence was associated with suicidality $(\mathrm{AOR}=3.18,95 \% \mathrm{CI}=1.79-5.67)$.
\end{abstract}

\section{Conclusions}

Adolescent health programs in India need to consider interventions for preventing adolescent suicidality, self-harm and depression that include improving parent-child relationships, reducing early marriage and preventing domestic violence.

Recent evidence on adolescent mortality documents that self-harm is the second leading cause of death for girls and the third leading cause of death for boys, globally. ${ }^{1}$ Selfharm and suicide, considered inter-related behaviors, ${ }^{2}$ are critical to adolescent mental health and development. ${ }^{3}$ Despite similar rates of suicidal ideation in high-income countries (HICs) and low- and middle-income countries (LMICs), ${ }^{4}$ research on risk factors and preventive efforts for suicide and self-harm among adolescents, have received far greater attention in HICs relative to LMICs. India has the largest population of adolescents of any nation in the world, ${ }^{5}$ as well as a disproportionate burden of suicides 6 making research directed towards adolescent suicide and self-harm in India not only necessary but important in combatting adolescent health disparities in LMICs.

In India, the past five years have seen calls to advance work on these issues, with a growing number of research studies that seek to examine gaps and drivers of mental 
health among young people in LMICs. ${ }^{7-9}$ India's national adolescent health strategy, the Rashtriya Kishor Swasthya Karyakram (RKSK), was also launched in 2014 with a mandate directed at expanding youth services to include mental health, gender-based violence and non-communicable diseases, with a focus on communication and prevention approaches that grounded adolescent agency within a framework of parents and the community. ${ }^{10,11}$ One such example of this growing body of literature includes a recent study on suicide mortality which showed elevated suicide death rates among later adolescent (15-19 years) and young adult (20-29 years) women. ${ }^{6}$ Few studies however have focused on adolescents let alone the different adolescent groups (unmarried or married early, middle or late aged adolescent girls or boys) to understand the specific drivers of adolescent suicidality in India following the RKSK. ${ }^{12}{ }^{13}$ Limited recent evidence on adolescents in India suggests an upward trend in adolescent self-harm/suicidality, and relatedly depression, ${ }^{14}$ and that family conflict may be compromising adolescent mental health and safety. ${ }^{14,15}$ Experiences of gender based violence are believed to operate within social settings marked by economic and social inequalities (in income, education, religion and ethnicity and residential context) that may set norms for acceptability of violence as a response or form of punishment. In India, more research is needed to understand the role of these socioeconomic factors in family violence research. Physical punishment of children as a form of discipline remains normative in the country, ${ }^{16}$ as does marital violence and children's witnessing of such violence. ${ }^{17}$ Adolescent marriage, a concern for about one in four adolescent girls in India, ${ }^{17}$ has been found to increase the girls' vulnerability for marital violence. ${ }^{17,18}$ Marital violence has furthermore been identified as a key risk factor for female reports of depression and suicidality among women across national settings, ${ }^{19}$ though it has not specifically been examined among married adolescent girls in India.

This study seeks to provide greater insight into the issue of family violence and risk for suicidality, self-harm and depression among adolescents in India. Given the gendered and developmental aspects noted above, analyses were stratified by sex (male and female) and age, $10-13,15-18,20$ with a separate focus on married girls and their direct experiences of marital violence. Due to the absence of research examining adolescent mental health in India, especially among younger adolescents and married girls, our study adds to the already established gender-based violence and mental health research while simultaneously increasing scholarship on adolescent mental health in LMICs. Findings from this work are expected to inform understandings of adolescents' health needs and ongoing gaps ${ }^{21}$ while supporting India's flagship adolescent health strategy, RKSK. $^{20}$

\section{METHODS}

\section{DATA AND SAMPLE}

We used data from the Understanding the lives of Adolescents and Young Adults (UDAYA) study conducted among 10-19 year olds in Bihar and Uttar Pradesh (UP), India. ${ }^{22,23}$ The
UDAYA study was led by the Population Council (New Delhi) with support from the Bill and Melinda Gates Foundation and the David and Lucile Packard Foundation and the Ministry of Health and Family Welfare (MOHFW), Government of India. The UDAYA study included cross-sectional and longitudinal surveys; in this study, we used data from the cross-sectional survey. Collected from September 2015 to July 2016, the cross-sectional survey sampled unmarried boys (10-19 years), unmarried girls (10-19 years), and married girls (15-19 years) in Uttar Pradesh (UP) and Bihar, India. This resulted in a sample total of 20,594 adolescents from Bihar $(n=10,433)$ and UP $(n=10,161)$, inclusive of 2,084 younger adolescent boys (10-14 years), 3,885 older adolescent boys (15-19 years), 1,653 younger adolescent girls (10-14 years), 7,766 older unmarried adolescent girls (15-19 years) and 5,206 older married adolescent girls (15-19 years).

\section{STUDY PROCEDURES FOR DATA COLLECTION}

Data in the cross-sectional survey were collected using a multi-stage systematic sampling approach, with three stage sampling in rural areas and four stage sampling in urban areas. ${ }^{22,23}$ Sampling frames come from the 2011 Census. Estimates from the UDAYA study are representative for the states in the study (as a whole) and for each respondent category in rural and urban areas; inferences cannot be made at district or sub-district levels. Region, village/ward size, Scheduled Caste (SC)/ Scheduled Tribe (ST) proportions and female literacy were used as selection criteria and samples were selected using probability proportional to size (PPS) methods. Adolescents 10-19 years (except divorced/widowed girls) were eligible for study inclusion. ${ }^{22}$ Interviewers were trained by experts prior to data collection to ensure specific technical modules (ie, the Patient Health Questionnaire or PHQ-9), were administered accurately to participants. Study instruments were extensively reviewed, translated into Hindi and finalized after pre-testing. Questionnaires were piloted to ensure that questions were relevant and age appropriate, and to minimize chances for error in survey implementation. Interviews were conducted using computer-assisted personal interview (CAPI) method in CSPro6.1. Response rates were high for interviews of boys (90-93\%) and girls (92-94\%); no differences were reported by residence. ${ }^{22,23}$

\section{ETHICS}

Details on ethical challenges faced by investigators, including consent, privacy and handling of sensitive subjects are available in the study reports. ${ }^{22,23}$ Adolescent boys and girls were interviewed in different locations to avoid any teasing or harassment. Age appropriate questions were asked to the respondents in non-judgmental and empathetic ways, with adolescents themselves contributing to instrument development on sensitive topics. Study investigators also sought consent from community leaders and for those below 18 years of age, from parents and guardians. All data were de-identified to maintain respondent confidentiality. Data were accessed through the Harvard Dataverse public data portal; this study received approval for ethical 
exemption from University of California, San Diego's institutional review board for research.

\section{MEASURE}

\section{DEPENDENT VARIABLES: SUICIDALITY, SELF-HARM AND} DEPRESSIVE SYMPTOMS

We considered three main dependent variables - suicidal ideation, self-harm behavior and depression.

For suicidal ideation, adolescents over the age of 13 years were asked, "During the past one year, did you ever seriously consider attempting suicide?" Response categories included "yes", "no" and "I don't want to answer". We categorized responses as No (reference) versus Yes, with “I don't want to answer" set to missing.

For self-harm behaviors, all adolescents $10-13,15-18,20$ were asked if when they were "agitated, angry or sad" in the past 12 months they had engaged in any self-harm behaviors, including: "cut or bit themselves", "pulled their own hair", and/or "banged or hit themselves". Responses were "never", "1-2 times", "3-4 times" or " $5+$ times" and were coded as No versus Yes (including the number of times). Additionally, if respondents reported any suicide attempts assessed by the question, "during the past one year, how many times did you actually attempt suicide”, these were included as Yes.

For depressive symptoms, we considered Patient Health Questionnaire-9 (PHQ-9) ${ }^{24}$ as an outcome and Patient Health Questionnaire-2 (PHQ-2) as a covariate. PHQ-9 is a standard depression screening instrument used globally and covers a range of depressive symptoms including feeling hopeless, trouble with sleep, lack of energy or pleasure in activities and concentration over the past 15 days or 2-week period. Likert scale responses options included "not at all 1 ", "less than one week ${ }^{2}$ ", "one week or more 3 " and "nearly every day". We recoded these responses as 0-3, and created a summary depressive symptoms score (range: 0 -27), categorized as "none" (0), "minimal depressive symptoms", 1-4 "mild depressive symptoms",5-9 "moderate depressive symptoms",10_13 "moderately severe depressive symptoms"14-18 and "severe depressive symptoms."19,21-27 Due to small cell sizes (ie, limited or no observations), we recoded the six category depression measure into three categories $(0=$ none; 1 =minimal-mild depressive symptoms; and $2=$ moderate-severe depressive symptoms). For boys $10-14$, we coded this variable as "any symptoms" and "no symptoms" due to limited symptomatology as only 1 person reported moderately severe depression, and none reported moderate depression and severe depression. As suicide is one of the items on the PHQ-9, the measure PHQ-9 could not be used as a covariate in our analyses of suicidality due to concerns of collinearity with the overlapping item. As a result, we used PHQ-2, a two-item depression screening measure that assessed depressed mood and anhedonia as our measure for depressive symptoms in models assessing suicidal ideation and self-harm. Initial scores ranged from $0-6$, and a cutoff of 3 was applied as recommended by prior research ${ }^{25}$ to create a dichotomous depression measures.

\section{INDEPENDENT VARIABLES: MEASURES OF FAMILY VIOLENCE}

Family violence was examined by three measures- witnessed parental marital violence, experienced child abuse, and personal experience of marital violence - with a focus on physical violence for specificity. Witnessing marital violence was assessed by the question, "Has your father ever beaten your mother?" Response options included "yes", "no", "don't know", and "not applicable". We created a binary yes/no measure, considering “don't know” and "not applicable” as missing. Child abuse was assessed by the question, "Have you been physically hurt (for example, beaten) by your father or mother from the time you turned 10 years old?" Response options included “yes”, “no”, and "not applicable”. We created a binary yes/no measure. Personal experience of marital violence was assessed for married girls using the question: "Did your husband ever do the following to you?" Items (rated yes/no) included "slap you”, "twist your arm or pull your hair"; "push you, shake you or throw something at you", "punch you with his fist or with something that could hurt you"; "kick you, drag you or beat you up"; "try to choke you or burn you on purpose"; "threaten or attack you with a knife, gun or any other weapon". We created a summation score where girls who said "yes" to any item were coded as having experienced marital violence.

\section{STRATIFICATION VARIABLES AND COVARIATES: SOCIO- DEMOGRAPHICS}

We used age categories (10-14 years, $15-19$ years), sex, and marital status as stratification variables. This stratification followed the structure of the sampling design and was used to ensure adolescent sub-population representativeness. Social equity variables along with individual and family socioeconomic status variables were considered in the analyses. Covariates represented a range of influencers including adolescent agency (schooling), parental and contextual factors (mother's education, residence, state), kinship and cultural influencers (religion and caste), economic factors (wealth) and health determinants of suicidality (depressive symptoms). For married girls, we additionally adjusted for age at marriage.

School enrollment was assessed from the question, "Are you currently attending a school or college?" Responses included "yes", "no", and "attending correspondence school". Due to limited response variability (0.03-6\% across respondents), we categorized attending correspondence school as "no" in the analyses.

Maternal education was assessed in the household questionnaire where the head of the household or an adult household member was asked about the maximum number of years an adolescent's mother spent attending school. Values were reclassified as follows: 0 years (including 'don't know'), 1-7 years, 8-9 years, and 10 or more years.

Religion was reported by the head of the household. Response options included "Hindu", "Muslim", "Christian", "Buddhist”, and "Others.” This was re-categorized as Hindu or Other Religions. 
Caste was reported by the head of the household. The variable included categories of "General Caste", "Scheduled caste (SC) or Scheduled Tribe (ST)" and "Other backward classes (OBCs)".

Residential area was classified as urban versus rural.

Wealth as a marker of household socioeconomic status was measured using data on household assets, durable goods (eg, television), means of transport and amenities such as cooking fuel, drinking water and electricity. Assets and amenities were assigned weights (total wealth index ranging from 0-57) and ranked households were categorized into quintiles. Quintile 1 (Q1) represented the poorest and quintile $5(\mathrm{Q} 5)$ represented the wealthiest household. ${ }^{22,23}$

State was Bihar or Uttar Pradesh (UP).

Age at marriage was assessed among married girls. The continuous variable (in years) was recoded as married at 18-19 years, married at $16-17$, and married before 16 years of age. This was done to distinguish very young marriages (before 16 years) versus those after 18 years or per the legal age at marriage.

\section{DATA ANALYSES}

We stratified all analyses by gender, age, and marital status, yielding five groups: boys 10-14, boys 15-19, girls 10-14, unmarried girls 15-19, and married girls 15-19. This stratification followed the study design and was considered due to the global interest on adolescent health issues from a gender and developmental perspective. Survey specific weights were used to ensure estimates were representative of the respondent populations of interest. ${ }^{22,23}$ We reported descriptive statistics for all variables, and conducted $\chi^{-2}$ tests to assess bivariate associations between family violence variables or measured covariates and our outcomes. Adjusted logistic regression models examined the associations between family violence and suicidality (with and without depressive symptoms as a covariate). Adjusted multinomial models were also estimated to examine the association between measures of family violence and depressive symptoms; among boys 10-14 years, logistic regression models were used for the dichotomized outcome due to small cell sizes. All analyses were conducted using STATA 15 (Stata Corp, College Station, TX, USA) and findings have been reported at 0.05 level of statistical significance.

\section{RESULTS}

This study is among the first to report prevalence of suicidality and rates of family violence among adolescents in India. One in twenty adolescents age $10-14$ years (5.35-6.07\%) and 15-19-year-old boys and 15-19 year old married girls (4.21-4.95\%) reported self-harm behaviors; nearly one in twenty unmarried adolescent girls also reported suicidal ideation (4.44\%) and this rate doubled (8.18\%) among married adolescent girls (Table 1). Among older adolescent girls, one in twenty (4.8\%) reported moderate to severe depressive symptoms, which doubled among married girls (8.15\%). Reports of moderate to severe depressive symptoms were low among 10-14 year old boys (0.03\%) and girls $(0.98 \%)$ and $15-19$ year old boys (1.77\%). Between 20.27-28.7\% of adolescents reported witnessing marital vi- olence among parents. Seven out of ten boys 10-14 reported child abuse, while one in two girls reported child abuse. Nearly one in four married $15-19$ year old girls (24.9\%) reported personal experience of marital violence.

\section{FAMILY VIOLENCE AND SUICIDAL IDEATION}

Bivariate analyses show that witnessing parental marital violence was associated with higher reports of suicidal ideation among 13-19 year old boys $(P<0.0001)$ and unmarried girls, and 15-19 year old married girls (Table S1 in the Online Supplementary Document). Child abuse was positively associated with suicidality among boys 13-19 years old $(P=0.0279)$ as well as among $13-19$ year old unmarried girls $(P<0.0001)$. Personal experience of marital violence and age at marriage were associated with suicidal ideation ( $P<0.0001$ and $P=0.0465$ respectively) among married adolescent girls (Table S1 in the Online Supplementary Document). In multivariate analyses, witnessing parental marital violence was associated with higher suicidal ideation among boys 13-19 years old (adjusted odds ratio $(\mathrm{AOR})=3.18,95 \%$ confidence intervals $(\mathrm{CI})=1.79-5.67)]$ and among married girls $15-19$ years old ( $\mathrm{AOR}=2.33$, 95\% $\mathrm{CI}=1.10-4.94$ ) (Table 2). Child abuse was associated with higher suicidal ideation among unmarried girls 13-19 years old ( $\mathrm{AOR}=2.08$, 95\% CI=1.40-3.07). Observed effects remain largely unchanged with the addition of a depression covariate to study models. Depression was associated with higher reports of suicidal ideation among boys 13-19 years old (AOR=3.08, 95\% CI=1.15-8.24), unmarried girls 13-19 years old $(\mathrm{AOR}=5.34,95 \% \mathrm{CI}=3.37-8.47)$ and married girls ( $\mathrm{AOR}=5.25,95 \% \mathrm{CI}=3.07-8.96)$. Among married girls, personal experience of marital violence was associated with higher reports of suicidal ideation $\mathrm{AOR}=3.98,95 \%$ $\mathrm{CI}=2.19-7.23)$; this effect was attenuated by depression (AOR=3.73, 95\% CI=2.00-6.96).

\section{FAMILY VIOLENCE AND SELF-HARM}

Witnessing parental marital violence was associated with higher reports of self-harm behaviors among boys 10-14 $(P=0.0043)$ and $15-19(P=0.0154)$ year olds, while experiencing child abuse was positively associated with self-harm behaviors among boys $10-14(P=0.002)$ and $15-19(P=0.0017)$ and unmarried girls $15-19(P<0.0001)$ (Table S2 in the Online Supplementary Document). Personal experience of marital violence and age at marriage were associated with self-harm behaviors ( $P=0.0003$ and $P=0.0161$, respectively) among married adolescent girls (Table S2 in the Online Supplementary Document). In multivariate models, measures of family violence were associated with increased reports of self-harm behaviors among boys 10-14 [witnessing parental marital violence $(\mathrm{AOR}=2.35,95 \% \mathrm{CI}=1.24-4.47)$ and experienced child abuse (AOR $=2.51,95 \% \mathrm{CI}=1.17-5.35)$ (Table 3). Child abuse was found to be associated with greater self-harm behaviors among 15-19 year old boys $(\mathrm{AOR}=2.22,95 \% \mathrm{CI}=1.27-3.89)$ and girls $(\mathrm{AOR}=2.79,95 \%$ $\mathrm{CI}=1.83-4.26$ ). Among married 15-19 year old girls, personal experiences of marital violence were associated with higher reports of self-harm behaviors $(\mathrm{AOR}=2.35, \quad 95 \%$ $\mathrm{CI}=1.16-4.75$ ), after adjusting for depression, this effect was 
Table 1. Study characteristics and measures of suicidal ideation, self-harm behavior, depression and family violence among adolescents (10-19 years) in the UDAYA study

\begin{tabular}{|c|c|c|c|c|c|c|}
\hline & $\begin{array}{l}\text { Boys } \\
10-14 \\
(n=2084)\end{array}$ & $\begin{array}{l}\text { Girls } \\
10-14 \\
(n=1653)\end{array}$ & $\begin{array}{l}\text { Boys } \\
15-19 \\
(n=3885)\end{array}$ & $\begin{array}{l}\text { Girls } 15-19, \\
\text { unmarried } \\
(n=7766)\end{array}$ & $\begin{array}{l}\text { Girls } 15-19, \\
\text { married } \\
(n=5206)\end{array}$ & $P$-value \\
\hline \multicolumn{7}{|c|}{ Socio-demographic characteristics } \\
\hline \multicolumn{7}{|c|}{ Current school enrollment } \\
\hline $\begin{array}{l}\text { No, including } \\
\text { correspondence }\end{array}$ & $114(6.0)$ & $\begin{array}{c}126 \\
(8.64)\end{array}$ & $\begin{array}{c}1026 \\
(29.37)\end{array}$ & $25161(39.58)$ & 3255 (88.92) & \multirow[t]{2}{*}{$<0.0001$} \\
\hline Yes & $\begin{array}{l}1923 \\
(94.0)\end{array}$ & $\begin{array}{l}1456 \\
(91.3)\end{array}$ & $\begin{array}{l}2703 \\
(70.6)\end{array}$ & $4698(60.4)$ & $529(11.0)$ & \\
\hline \multicolumn{7}{|l|}{ State of residence } \\
\hline UP & $\begin{array}{c}1072 \\
(65.14)\end{array}$ & $\begin{array}{c}889 \\
(65.0)\end{array}$ & $\begin{array}{c}2064 \\
(69.79)\end{array}$ & $4338(71.62)$ & $1798(61.82)$ & \multirow[t]{2}{*}{$<0.0001$} \\
\hline Bihar & $\begin{array}{c}1012 \\
(34.86)\end{array}$ & $\begin{array}{c}764 \\
(35.0)\end{array}$ & $\begin{array}{c}1821 \\
(30.21)\end{array}$ & $3428(28.38)$ & 3408 (38.18) & \\
\hline \multicolumn{7}{|l|}{ Area of residence } \\
\hline Urban & $\begin{array}{c}872 \\
(16.48)\end{array}$ & $\begin{array}{c}759 \\
(17.02)\end{array}$ & $\begin{array}{c}1960 \\
(17.67)\end{array}$ & $3746(17.31)$ & $1942(14.03)$ & \multirow[t]{2}{*}{$<0.0001$} \\
\hline Rural & $\begin{array}{c}1212 \\
(83.52)\end{array}$ & $\begin{array}{c}894 \\
(82.98)\end{array}$ & $\begin{array}{c}1925 \\
(82.33)\end{array}$ & $4020(82.69)$ & $3264(85.97)$ & \\
\hline \multicolumn{7}{|l|}{ Mother's education } \\
\hline 0 years & $\begin{array}{l}1394 \\
(72.2)\end{array}$ & $\begin{array}{c}1102 \\
(70.11)\end{array}$ & $\begin{array}{c}2476 \\
(70.23)\end{array}$ & $5082(70.41)$ & $4327(82.97)$ & \multirow[t]{4}{*}{$<0.0001$} \\
\hline $1-7$ years & $216(9.19)$ & $\begin{array}{c}195 \\
(13.15)\end{array}$ & $\begin{array}{c}432 \\
(10.91)\end{array}$ & $841(10.43)$ & $427(8.62)$ & \\
\hline $8-9$ years & $191(8.76)$ & $\begin{array}{c}146 \\
(8.03)\end{array}$ & $350(7.9)$ & $644(7.88)$ & $240(4.63)$ & \\
\hline $10+$ years & $283(9.85)$ & $\begin{array}{c}210 \\
(8.72)\end{array}$ & $\begin{array}{c}627 \\
(10.96)\end{array}$ & 1199 (11.28) & $212(3.77)$ & \\
\hline \multicolumn{7}{|l|}{ Religion } \\
\hline Hindu & $\begin{array}{c}1712 \\
(83.77)\end{array}$ & $\begin{array}{c}1263 \\
(77.57)\end{array}$ & $\begin{array}{c}3196 \\
(83.78)\end{array}$ & $5636(75.98)$ & 4391 (82.52) & \multirow[t]{2}{*}{$<0.0001$} \\
\hline Other religions & $\begin{array}{c}372 \\
(16.23)\end{array}$ & $\begin{array}{c}390 \\
(22.43)\end{array}$ & $\begin{array}{c}689 \\
(16.22)\end{array}$ & $2130(24.02)$ & 815 (17.48) & \\
\hline \multicolumn{7}{|l|}{ Caste } \\
\hline General & $\begin{array}{c}407 \\
(18.37)\end{array}$ & $\begin{array}{c}338 \\
(18.69)\end{array}$ & $\begin{array}{c}782 \\
(18.15)\end{array}$ & $1805(22.2)$ & $526(12.61)$ & \multirow[t]{3}{*}{0.0001} \\
\hline $\begin{array}{l}\text { Scheduled caste/ } \\
\text { scheduled Tribe (SC/ } \\
\text { ST) }\end{array}$ & $\begin{array}{c}493 \\
(26.13)\end{array}$ & $\begin{array}{c}365 \\
(24.84)\end{array}$ & $\begin{array}{c}912 \\
(27.46)\end{array}$ & $1520(22.8)$ & $1464(29.06)$ & \\
\hline $\begin{array}{l}\text { Other backward } \\
\text { classes (OBC) }\end{array}$ & $\begin{array}{l}1184 \\
(55.5)\end{array}$ & $\begin{array}{c}950 \\
(56.47)\end{array}$ & $\begin{array}{c}2191 \\
(56.39)\end{array}$ & $4441(55.0)$ & $3216(58.33)$ & \\
\hline \multicolumn{7}{|l|}{ Wealth quintile } \\
\hline Q1 (poorest) & $\begin{array}{c}278 \\
(16.64)\end{array}$ & $\begin{array}{c}218 \\
(16.6)\end{array}$ & $278(9.18)$ & 723 (12.08) & $860(14.57)$ & \multirow[t]{5}{*}{$<0.0001$} \\
\hline Q2 (poorer) & $\begin{array}{c}376 \\
(21.91)\end{array}$ & $\begin{array}{c}273 \\
(19.8)\end{array}$ & $\begin{array}{c}536 \\
(18.95)\end{array}$ & $1017(17.24)$ & $1022(20.53)$ & \\
\hline Q3 (middle) & $\begin{array}{c}432 \\
(21.58)\end{array}$ & $\begin{array}{c}310 \\
(20.13)\end{array}$ & $\begin{array}{c}777 \\
(23.78)\end{array}$ & 1438 (21.03) & 1239 (23.47) & \\
\hline Q4 (richer) & $\begin{array}{c}476 \\
(19.92)\end{array}$ & $\begin{array}{c}446 \\
(24.03)\end{array}$ & $\begin{array}{c}1124 \\
(25.11)\end{array}$ & $2142(24.7)$ & $1289(24.23)$ & \\
\hline Q5 (richest) & $\begin{array}{c}522 \\
(19.94)\end{array}$ & $\begin{array}{c}406 \\
(19.44)\end{array}$ & $\begin{array}{c}1170 \\
(22.97)\end{array}$ & $2446(24.95)$ & $796(17.2)$ & \\
\hline \multicolumn{7}{|l|}{ Age at marriage } \\
\hline $18-19$ years & NA & NA & NA & NA & $1230(32.21)$ & \\
\hline $16-17$ years & NA & NA & NA & NA & $2280(45.32)$ & \\
\hline
\end{tabular}




\begin{tabular}{|c|c|c|c|c|c|c|}
\hline & $\begin{array}{l}\text { Boys } \\
10-14 \\
(n=2084)\end{array}$ & $\begin{array}{l}\text { Girls } \\
10-14 \\
(n=1653)\end{array}$ & $\begin{array}{l}\text { Boys } \\
15-19 \\
(n=3885)\end{array}$ & $\begin{array}{l}\text { Girls 15-19, } \\
\text { unmarried } \\
(n=7766)\end{array}$ & $\begin{array}{l}\text { Girls 15-19, } \\
\text { married } \\
(n=5206)\end{array}$ & P-value \\
\hline$<16$ years & NA & NA & NA & NA & $1383(22.47)$ & \\
\hline \multicolumn{7}{|c|}{ Measures of family violence } \\
\hline \multicolumn{7}{|c|}{ Witnessed marital violence among parents } \\
\hline No & $\begin{array}{c}1454 \\
(76.47)\end{array}$ & $\begin{array}{c}1126 \\
(71.28)\end{array}$ & $\begin{array}{c}2919 \\
(79.73)\end{array}$ & $5541(74.23)$ & $3390(68.1)$ & \multirow[t]{2}{*}{$<0.0001$} \\
\hline Yes & $\begin{array}{c}407 \\
(23.53)\end{array}$ & $\begin{array}{c}423 \\
(28.72)\end{array}$ & $\begin{array}{c}631 \\
(20.27)\end{array}$ & $1788(25.77)$ & 1537 (31.9) & \\
\hline \multicolumn{7}{|c|}{ Experienced direct parental abuse since age 10} \\
\hline No & $\begin{array}{c}644 \\
(29.43)\end{array}$ & $\begin{array}{c}797 \\
(48.66)\end{array}$ & $\begin{array}{c}1729 \\
(45.83)\end{array}$ & $5222(67.47)$ & $3520(68.36)$ & \multirow[t]{2}{*}{$<0.0001$} \\
\hline Yes & $\begin{array}{c}1392 \\
(70.57)\end{array}$ & $\begin{array}{c}798 \\
(51.34)\end{array}$ & $\begin{array}{c}1970 \\
(54.17)\end{array}$ & $2254(32.53)$ & $1602(31.64)$ & \\
\hline \multicolumn{7}{|c|}{ Own experience of marital violence } \\
\hline No & NA & NA & NA & NA & $3552(75.06)$ & \\
\hline Yes & NA & NA & NA & NA & $1349(24.94)$ & \\
\hline \multirow{2}{*}{\multicolumn{7}{|c|}{$\begin{array}{l}\text { Suicidal ideation, self-harm and depression } \\
\text { Suicidal ideation } \ddagger\end{array}$}} \\
\hline & & & & & & \\
\hline No & $\begin{array}{c}768 \\
(99.17)\end{array}$ & $\begin{array}{c}687 \\
(98.88)\end{array}$ & $\begin{array}{c}3769 \\
(97.56)\end{array}$ & $7397(95.56)$ & $4833(91.82)$ & \multirow[t]{2}{*}{$<0.0001$} \\
\hline Yes & $6(0.83)$ & $10(1.12)$ & $87(2.44)$ & $335(4.44)$ & $351(8.18)$ & \\
\hline \multicolumn{7}{|l|}{ Self-harm } \\
\hline No & $\begin{array}{c}1984 \\
(94.65)\end{array}$ & $\begin{array}{c}1560 \\
(93.93)\end{array}$ & $\begin{array}{c}3719 \\
(95.79)\end{array}$ & 7500 (97.09) & $4999(95.05)$ & \multirow[t]{2}{*}{0.0005} \\
\hline Yes & $100(5.35)$ & $93(6.07)$ & $166(4.21)$ & $266(2.91)$ & $207(4.95)$ & \\
\hline \multicolumn{7}{|c|}{ Depressive symptoms (PHQ-9 categories)§ } \\
\hline No & $\begin{array}{c}1430 \\
(69.72)\end{array}$ & $\begin{array}{c}1146 \\
(70.25)\end{array}$ & $\begin{array}{c}2392 \\
(60.84)\end{array}$ & $4255(54.71)$ & $2580(48.73)$ & \multirow[t]{3}{*}{$<0.0001$} \\
\hline Minimal-mild & $\begin{array}{c}653 \\
(30.23)\end{array}$ & $\begin{array}{c}484 \\
(28.76)\end{array}$ & $\begin{array}{c}1429 \\
(37.38)\end{array}$ & $3092(40.40)$ & $2234(43.11)$ & \\
\hline Moderate-severe & $1(0.03)$ & $23(0.98)$ & $64(1.77)$ & $419(4.88)$ & $392(8.15)$ & \\
\hline \multicolumn{7}{|c|}{ Depressive symptoms screening (PHQ-2)T } \\
\hline$<3$ & $\begin{array}{c}2071 \\
(99.38)\end{array}$ & $\begin{array}{c}1624 \\
(98.97)\end{array}$ & $\begin{array}{c}3778 \\
(97.26)\end{array}$ & $7254(93.74)$ & $4779(91.34)$ & \multirow[t]{2}{*}{$<0.0001$} \\
\hline $3+$ & $13(0.62)$ & $29(1.03)$ & $107(2.74)$ & $512(6.26)$ & $427(8.66)$ & \\
\hline
\end{tabular}

Note: (table presents distributions and weighted proportions with $P$-value for differences)

NA - not applicable

"For maternal education, variable in the data combined 'Don't know' with 'none' and for current schooling, small number of correspondence frequencies were pooled with No $* * * * *$ Suicidal ideation was asked of adolescents 13 years and over; hence the categories of adolescents 10-14 include responses by 13 and 14 year olds only

§Depressive symptoms as measured by patient health questionnaire (PHQ-9) and used as a dependent variable was categorized as none (PHQ-9=0), Mild $(1<\mathrm{PHQ}-9<9)$ and ModerateSevere (10<PHQ-9<27).

IDepressive symptoms screening as measured by Patient Health Questionnaire (PHQ-2) was used as a covariate in the analyses for suicide and self-harm behavior. Scores ranges from $0-6$, with a score of 3 as the cut point for likelihood of major depressive disorder.

no longer statistically significant. Depression (AOR=5.42, 95\% $\mathrm{CI}=3.29-8.93)$ and age at marriage $(\mathrm{AOR}=2.71,95 \%$ $\mathrm{CI}=1.10-6.63)$ were also associated with increased selfharm behaviors.

\section{FAMILY VIOLENCE AND DEPRESSIVE SYMPTOMS}

Witnessing parental marital violence was associated with higher reports of mild depressive symptoms among boys $(15-19 ; P=0.0006)$ and married girls $(15-19 ; P<0.0001)$, and higher reports of moderate to severe depressive symptoms among unmarried girls $(15-19 ; \mathrm{P}<0.01)$ and married girls
(15-19; $P=0.0006)$ (Table S3 in the Online Supplementary Document). Moreover, child abuse was associated with mild depressive symptoms among 15-19 year old boys $(P=0.0061)$, and among unmarried $(P=0.001)$ and married 15 - 19 year old girls $(P=0.055)$. In multivariate analyses, witnessing marital violence was associated with the increased endorsement of mild depressive symptoms among girls 10-14 year old $(\mathrm{AOR}=1.69,95 \% \mathrm{CI}=1.12-2.54)$, boys $15-19$ years old $(A O R=1.51,95 \% \mathrm{CI}=1.18-1.93)$ and married girls $15-19$ years old $(\mathrm{AOR}=1.52,95 \% \mathrm{CI}=1.09-2.09)$ (Table 4$)$. For unmarried girls $15-19$ years old, witnessing marital violence was associated with an increased likelihood for moderate to 
Table 2. Multivariate association (odds ratios, OR (95\% CIs)) between measures of family violence and suicidal ideation (categorized as No versus Yes) among adolescents (13-19 years) in the UDAYA study in India*

\begin{tabular}{|c|c|c|c|c|c|c|}
\hline & $\begin{array}{l}\text { Boys } \\
13-19 \\
(n=3938)\end{array}$ & $\begin{array}{l}\text { Girls 13-19, } \\
\text { unmarried } \\
(n=7224)\end{array}$ & $\begin{array}{l}\text { Girls 15-19, } \\
\text { married } \\
(n=4596)\end{array}$ & & & \\
\hline & $\begin{array}{l}\text { M1: OR } \\
(95 \% \mathrm{CI})\end{array}$ & $\begin{array}{l}\text { M2: OR (95\% } \\
\text { CI) }\end{array}$ & $\begin{array}{l}\text { M1: OR (95\% } \\
\text { Cl) }\end{array}$ & $\begin{array}{l}\text { M2: OR } \\
(95 \% \mathrm{CI})\end{array}$ & $\begin{array}{l}\text { M1: OR } \\
(95 \% \mathrm{Cl})\end{array}$ & $\begin{array}{l}\text { M2: OR } \\
(95 \% \mathrm{Cl})\end{array}$ \\
\hline \multicolumn{7}{|c|}{ Witnessed marital violence among parents (Ref: No) } \\
\hline Yes & $\begin{array}{c}3.22 \dagger \\
(1.83-5.70)\end{array}$ & $\begin{array}{c}3.18^{*} \\
(1.79-5.67)\end{array}$ & $\begin{array}{c}1.55 \\
(0.99-2.41)\end{array}$ & $\begin{array}{c}1.52 \\
(0.98-2.36)\end{array}$ & $\begin{array}{c}2.33^{*} \\
(1.14-4.75)\end{array}$ & $\begin{array}{c}2.33^{*} \\
(1.10-4.94)\end{array}$ \\
\hline \multicolumn{7}{|c|}{ Experienced parental abuse since age 10 (Ref: No) } \\
\hline Yes & $\begin{array}{c}1.36 \\
(0.72-2.56)\end{array}$ & $\begin{array}{c}1.40 \\
(0.74-2.68)\end{array}$ & $\begin{array}{c}2.09^{*} \\
(1.42-3.08)\end{array}$ & $\begin{array}{c}2.08^{*} \\
(1.40-3.07)\end{array}$ & $\begin{array}{c}0.82 \\
(0.45-1.48)\end{array}$ & $\begin{array}{c}0.85 \\
(0.46-1.57)\end{array}$ \\
\hline \multicolumn{7}{|c|}{ Experience of marital violence (Ref: No) } \\
\hline Yes & NA & NA & NA & NA & $\begin{array}{c}3.98^{*} \\
(2.19-7.23)\end{array}$ & $\begin{array}{l}3.73^{*} \\
(2.00 \\
6.96)\end{array}$ \\
\hline \multicolumn{7}{|l|}{ Caste (Ref: General) } \\
\hline $\begin{array}{l}\text { Scheduled Caste/ } \\
\text { Scheduled Tribe } \\
\text { (SC/ST) }\end{array}$ & $\begin{array}{c}1.66 \\
(0.70-3.90)\end{array}$ & $\begin{array}{c}1.71 \\
(0.73-4.00)\end{array}$ & $\begin{array}{c}2.40^{*} \\
(1.22-4.75)\end{array}$ & $\begin{array}{c}2.43^{*} \\
(1.19-4.94)\end{array}$ & $\begin{array}{c}0.93 \\
(0.45-1.91)\end{array}$ & $\begin{array}{c}0.83 \\
(0.39-1.71)\end{array}$ \\
\hline $\begin{array}{l}\text { Other Backward } \\
\text { Classes (OBC) }\end{array}$ & $\begin{array}{c}1.03 \\
(0.44-2.37)\end{array}$ & $\begin{array}{c}1.08 \\
(0.47-2.49)\end{array}$ & $\begin{array}{c}1.73 \\
(0.97-3.08)\end{array}$ & $\begin{array}{c}1.85^{*} \\
(1.02-3.37)\end{array}$ & $\begin{array}{c}1.02 \\
(0.57-1.81)\end{array}$ & $\begin{array}{c}1.06 \\
(0.58-1.94)\end{array}$ \\
\hline \multicolumn{7}{|c|}{ Depression, categories of PHQ-2 (Ref: $<3) \dagger$} \\
\hline$\geq 3$ (major dep likely) & & $\begin{array}{c}3.08^{*} \\
(1.15-8.24)\end{array}$ & & $\begin{array}{c}5.34^{*} \\
(3.37-8.47)\end{array}$ & & $\begin{array}{c}5.25^{*} \\
(3.07-8.96)\end{array}$ \\
\hline \multicolumn{7}{|c|}{ Age at marriage, in years (Ref: $18-19$ years) } \\
\hline $16-17$ & NA & NA & NA & NA & $\begin{array}{c}1.006 \\
(0.62-1.64)\end{array}$ & $\begin{array}{c}0.96 \\
(0.60-1.54)\end{array}$ \\
\hline$<16$ & NA & NA & NA & NA & $\begin{array}{c}2.20 \\
(1.04-4.65)\end{array}$ & $\begin{array}{c}2.13 \\
(0.97-4.71)\end{array}$ \\
\hline
\end{tabular}

OR - odds ratio, CI - confidence interval, NA - not applicable, Ref - reference.

*ORs in bold and * represent $P<0.05$. M1 (model 1) adjusted for school enrollment, mother's education, residence area, state, religion, caste and wealth, while M2 (model 2), in addition adjusted for depressive symptoms. Among married girls, analyses also included own experience of marital violence and age at marriage. Categories for school enrollment considered correspondence school as No due to small number of adolescents reporting correspondence school.

†Depressive Symptom Screening patient health questionnaire PHQ-2 was used categorized as binary: details in the methods section.

severe depressive symptoms $(\mathrm{AOR}=1.81,95 \% \mathrm{CI}=1.30-2.50)$. Child abuse was also associated with an increased likelihood for mild depressive symptoms among boys 15-19 (AOR=1.27, 95\%CI=1.03- 1.57) and unmarried girls $15-19$ year old boys $(\mathrm{AOR}=1.47,95 \% \mathrm{CI}=1.24-1.74)$. Among married girls, marital violence was associated with an increased likelihood in reporting mild ( $\mathrm{AOR}=2.19,95 \% \mathrm{CI}=1.65-2.9)$ and moderate to severe depressive symptoms (AOR=2.93, 95\% CI=1.83- 4.68). Mild and moderate to severe symptomatology was assessed relative to no depressive symptomatology (reference group).

Supplemental analyses (Table S4 in the Online Supplementary Document) among married adolescent girls showed an association between witnessing parental marital violence and personal experience of marital violence (AOR=2.24, 95\% CI=1.69-2.96), as well as for child abuse (AOR=1.86, 95\% CI=1.48-2.34). Compared to girls who married at 18-19 years of age, marriage at 16-17 years of age and marriage before age 16 were associated with marital violence: marriage at $16-17$ years $(A O R=1.66,95 \%$ $\mathrm{CI}=1.17-2.36)$ and marriage at $<16$ years $(\mathrm{AOR}=2.98$, 95\% $\mathrm{CI}=2.15-4.15)$. School enrollment was associated with re- duced odds of experiencing marital violence (AOR: 0.32, $95 \% \mathrm{CI}=0.18$ - 0.54 ) among married girls $15-19$ years old.

\section{DISCUSSION}

Findings from this study add to the growing body of evidence suggesting that suicidality, self-harm and depression are major adolescent health problems in India. ${ }^{14}$ Consistent with previous research 26,27 we also find that these issues disproportionately affect older adolescents and girls, especially married girls. As expected, family and marital violence were associated with adolescent depression, selfharm and suicidality in India. Married adolescent girls appeared to be the most vulnerable to violence, facing violence both in their natal home and by their husband. This is in line with previous studies documenting the vulnerability of child brides to experiences of violence. ${ }^{28}$ Rates of marital violence in this study were comparable to the national prevalence estimates of intimate partner violence among currently married women since the age of 15 (33\% per recently released National Family Health Survey (NFHS) data) (Table 1). Parental physical abuse was also linked to an in- 
Table 3. Multivariate Associations (odds ratios, OR (95\% CIs)) between measures of Family Violence and Self-Harm Behavior (categorized as No versus Yes) among Adolescents (10-19 years) in the UDAYA study in India.

\begin{tabular}{|c|c|c|c|c|c|c|c|c|c|}
\hline & $\begin{array}{l}\text { Boys 10-14, } \\
(n=1795)\end{array}$ & $\begin{array}{l}\text { Girls 10-14, } \\
(\mathrm{n}=1445)\end{array}$ & $\begin{array}{l}\text { Boys 15-19, } \\
(\mathrm{n}=3285)\end{array}$ & $\begin{array}{l}\text { Girls } 15-19, \text { unmarried, } \\
(\mathrm{n}=6649)\end{array}$ & $\begin{array}{l}\text { Girls 15-19, married, } \\
(n=4613)\end{array}$ & & & & \\
\hline & $\begin{array}{l}\text { M1: OR }(95 \% \\
\text { CI) }\end{array}$ & $\begin{array}{l}\text { M1: OR (95\% } \\
\text { CI) }\end{array}$ & $\begin{array}{l}\text { M2: OR }(95 \% \\
\mathrm{Cl})\end{array}$ & M1: OR (95\% CI) & M2: OR (95\% Cl) & $\begin{array}{l}\text { M1: OR } \\
(95 \% \mathrm{Cl})\end{array}$ & $\begin{array}{l}\text { M2: OR } \\
(95 \% \mathrm{Cl})\end{array}$ & $\begin{array}{l}\text { M1: OR } \\
(95 \% \mathrm{Cl})\end{array}$ & $\begin{array}{l}\text { M2: OR } \\
(95 \% \mathrm{Cl})\end{array}$ \\
\hline \multicolumn{10}{|c|}{ Witnessed marital violence among parents (Ref: No) } \\
\hline Yes & $\begin{array}{c}2.35^{*} \\
(1.24-4.49)\end{array}$ & $\begin{array}{c}1.70 \\
(0.92-3.17)\end{array}$ & $\begin{array}{c}1.69 \\
(0.91-3.18)\end{array}$ & $1.40(0.77-2.56)$ & $1.40(0.77-2.56)$ & $\begin{array}{c}1.02 \\
(0.67-1.56)\end{array}$ & $\begin{array}{c}0.98 \\
(0.64-1.5)\end{array}$ & $\begin{array}{c}1.61 \\
(0.67-3.89)\end{array}$ & $\begin{array}{c}1.53 \\
(0.62-3.92)\end{array}$ \\
\hline \multicolumn{10}{|c|}{ Experienced parental abuse since age 10 (Ref: No) } \\
\hline Yes & $\begin{array}{c}2.51^{*} \\
(1.17-5.35)\end{array}$ & $\begin{array}{c}0.87 \\
(0.50-1.53)\end{array}$ & $\begin{array}{l}0.87(0.49- \\
1.53)\end{array}$ & $2.21^{*}(1.26-3.89)$ & $2.22^{*}(1.27-3.89)$ & $\begin{array}{l}2.77^{*}(1.84- \\
4.17)\end{array}$ & $\begin{array}{l}2.79 *(1.83- \\
\quad 4.26)\end{array}$ & $\begin{array}{c}0.80 \\
(0.39-1.64)\end{array}$ & $\begin{array}{c}0.83 \\
(0.39-1.72)\end{array}$ \\
\hline \multicolumn{10}{|c|}{ Experience of marital violence (Ref: No) } \\
\hline Yes & NA & NA & NA & NA & NA & NA & NA & $\begin{array}{c}2.35^{*} \\
(1.16-4.75)\end{array}$ & $\begin{array}{c}1.97 \\
(0.95-4.07)\end{array}$ \\
\hline \multicolumn{10}{|c|}{ Depression, categories of PHQ-2 (Ref: 0-2) $\ddagger$} \\
\hline$\geq 3$ & & & $\begin{array}{c}4.38^{*} \\
(1.21-15.91)\end{array}$ & & $1.43(0.4-5.05)$ & & $\begin{array}{c}5.1^{*} \\
(3.01-8.64)\end{array}$ & & $\begin{array}{c}5.42^{*} \\
(3.29-8.93)\end{array}$ \\
\hline \multicolumn{10}{|c|}{ Age at marriage, in years (Ref: $18-19$ years) } \\
\hline $16-17$ & NA & NA & NA & NA & NA & NA & NA & $\begin{array}{c}1.65 \\
(0.91-2.97)\end{array}$ & $\begin{array}{c}1.49 \\
(0.85-2.61)\end{array}$ \\
\hline$<16$ & NA & NA & NA & NA & NA & NA & NA & $\begin{array}{c}2.86^{*} \\
(1.22-6.81)\end{array}$ & $\begin{array}{c}2.71^{*} \\
(1.10-6.63)\end{array}$ \\
\hline
\end{tabular}

OR - odds ratio, CI - confidence interval, NA - not applicable Ref - reference.

Note: ORs in bold and * represent $P<0.05$. For girls $15-19$, a significant proportion of missing for the variable for current schooling was noted, and missing was considered as a separate category.

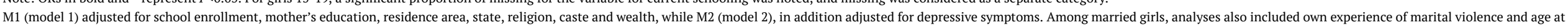
marriage. Categories for school enrollment considered correspondence school as No due to small number of adolescents reporting correspondence school.

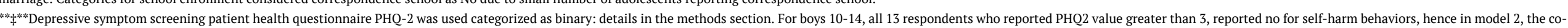
variate for depression dropped out and only M1 gives estimates. 
Table 4. Multivariate associations (relative risk ratios, RRRs (95\% CIs) between measures of family violence and depressive symptoms (none, mild and moderate-severe categories of PHQ-9) among adolescents (10-19 years) in the UDAYA study in India

\begin{tabular}{|c|c|c|c|c|c|c|c|c|c|}
\hline & \multirow{2}{*}{$\begin{array}{c}\text { Boys } \\
10-14 \dagger, \ddagger \\
\text { Any } \\
\text { Depression }\end{array}$} & \multicolumn{2}{|c|}{ Girls 10-14¥ } & \multicolumn{2}{|c|}{ Boys 15-19ł } & \multicolumn{2}{|c|}{ Girls 15-19, unmarried $\neq$} & \multicolumn{2}{|c|}{ Married girls (15-19 years)ł } \\
\hline & & $\begin{array}{l}\text { Mild } \\
\text { symptoms }\end{array}$ & $\begin{array}{c}\text { Moderate- } \\
\text { severe symptoms }\end{array}$ & $\begin{array}{l}\text { Mild } \\
\text { symptoms }\end{array}$ & $\begin{array}{l}\text { Moderate - } \\
\text { severe symptoms }\end{array}$ & $\begin{array}{l}\text { Mild } \\
\text { symptoms }\end{array}$ & $\begin{array}{c}\text { Moderate - } \\
\text { severe symptoms }\end{array}$ & $\begin{array}{c}\text { Mild } \\
\text { symptoms }\end{array}$ & $\begin{array}{c}\text { Moderate - } \\
\text { severe symptoms }\end{array}$ \\
\hline \multicolumn{10}{|c|}{ Witnessed marital violence among parents (Ref: No): } \\
\hline Yes & $\begin{array}{c}0.88 \\
(0.63-1.23)\end{array}$ & $\begin{array}{c}1.69^{*} \\
(1.12-2.54)\end{array}$ & $1.79(0.44-7.30)$ & $\begin{array}{c}1.51^{*} \\
(1.18-1.93)\end{array}$ & $1.02(0.40-2.61)$ & $\begin{array}{c}0.95 \\
(0.78-1.15)\end{array}$ & $1.81^{*}(1.30-2.50)$ & $\begin{array}{c}1.52^{*} \\
(1.09-2.09)\end{array}$ & $1.43(0.93-2.19)$ \\
\hline \multicolumn{10}{|c|}{ Experienced parental abuse since age 10 (Ref: No): } \\
\hline Yes & $\begin{array}{l}1.23(0.90- \\
1.67)\end{array}$ & $\begin{array}{c}1.11(0.77- \\
1.60)\end{array}$ & $0.66(0.20-2.16)$ & $\begin{array}{c}1.27^{*} \\
(1.03- \\
1.57)\end{array}$ & $0.87(0.40-1.85)$ & $\begin{array}{c}1.47^{*} \\
(1.24- \\
1.74)\end{array}$ & $1.11(0.77-1.61)$ & $\begin{array}{c}1.01(0.75- \\
1.36)\end{array}$ & $0.98(0.63-1.53)$ \\
\hline \multicolumn{10}{|c|}{ Experience of marital violence (Ref: No): } \\
\hline Yes & NA & NA & NA & NA & NA & NA & NA & $\begin{array}{c}2.19^{*} \\
(1.65-2.9)\end{array}$ & $2.93^{*}(1.83-4.68)$ \\
\hline \multicolumn{10}{|c|}{ Current school enrollment (Ref: No): } \\
\hline Yes & $\begin{array}{c}1.49 \\
(0.82-2.73)\end{array}$ & $\begin{array}{c}0.67 \\
(0.40-1.12)\end{array}$ & $0.31(0.05-1.84)$ & $\begin{array}{c}1.07 \\
(0.83-1.38)\end{array}$ & $0.60(0.29-1.25)$ & $\begin{array}{c}1.02 \\
(0.85-1.23)\end{array}$ & $0.79(0.54-1.16)$ & $\begin{array}{c}0.82 \\
(0.57-1.18)\end{array}$ & $1.22(0.53-2.83)$ \\
\hline Correspondence & & & & $\begin{array}{l}1.12(0.66- \\
1.89)\end{array}$ & $0.49(0.06-3.85)$ & $\begin{array}{c}1.43^{*} \\
(1.05- \\
1.94)\end{array}$ & $0.98(0.44-2.17)$ & $\begin{array}{c}1.46 \\
(0.97-2.19)\end{array}$ & $0.83(0.381 .-83)$ \\
\hline \multicolumn{10}{|c|}{ Religion: (Ref: Hindu): } \\
\hline Other religions & $\begin{array}{c}1.92^{*} \\
(1.29- \\
2.87)\end{array}$ & $\begin{array}{l}0.74(0.48- \\
1.15)\end{array}$ & $1.09(0.19-6.09)$ & $\begin{array}{c}1.03 \\
(0.71-1.48)\end{array}$ & $1.32(0.49-3.55)$ & $\begin{array}{c}1.06 \\
(0.87-1.29)\end{array}$ & $1.23(0.86-1.78)$ & $\begin{array}{c}1.36 \\
(0.97-1.92)\end{array}$ & $1.66(0.99-2.79)$ \\
\hline \multicolumn{10}{|c|}{ Age at marriage, in years (Ref: 18-19): } \\
\hline $16-17$ & NA & NA & NA & NA & NA & NA & NA & $\begin{array}{c}0.83 \\
(0.63-1.08)\end{array}$ & $0.89(0.52-1.51)$ \\
\hline$<16$ & NA & NA & NA & NA & NA & NA & NA & $\begin{array}{c}0.88 \\
(0.63-1.23)\end{array}$ & $1.50(0.73-3.08)$ \\
\hline
\end{tabular}

"OR - odds ratio, and CI - confidence interval (in bold and " represent $P<0.05$ ), others represented as relative risk ratio (RRR).

NA - not applicable, Ref - reference.

†For 10-14 year old boys, we used logistic regression models with the outcome any depressive symptoms (none versus any) as low frequencies in moderate to severe categorization. For other adolescent sub-groups, multinomial regression models were used.

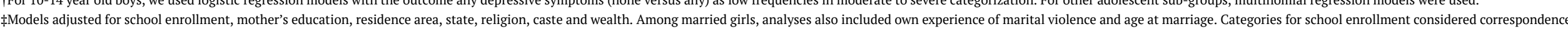
school as No due to small number of adolescents reporting correspondence school. 
creased risk for marital violence among girls 15-19 years old. These findings suggest a multi-generational pattern of influence as family violence appears to affect girls' propensity for future violence, suicidality or self-harm, and depression. Inequalities in education, caste, religion and wealth influenced suicidal ideation, self-harm and depression, particularly among older unmarried girls (Tables S1-S3 in the Online Supplementary Document).

High rates of witnessing parents' marital violence (20-31.9\%) were noted across adolescent subsamples. Rates of physical abuse were high (31.6-70.5\%), especially among boys aged 10-14. Prevalence of physical abuse was much higher among younger boys than for younger girls. One reason young boys report physical abuse at a higher rate than girls may be due to traditional gender norms or culturally based parenting practices. Girls in India tend to be socialized to be agreeable and conform to social expectations ${ }^{29,30}$ while boys are more able to easily challenge authority figures. ${ }^{29,30}$ This may result in boys "acting out" in ways that parents feel would warrant a harsher response. Authoritative parenting is more common in India than the US and does utilize punitive methods such as "hitting" or "beating" a child. ${ }^{31}$ Only a small subset of boys $10-14$ who reported being abused by parents endorsed depressive symptoms, though they did report suicidality. This discordant finding might be due to gender differences in depression, ${ }^{32,33}$ mental illness stigma, ${ }^{34}$ and societal expectations that boys should not be emotional. ${ }^{29,30}$

Depression associated with suicidality among adolescents has been supported by the global literature. ${ }^{3,35}$ The fact that reports of depressive symptoms were exceptionally low among younger adolescents, particularly boys, could be attributed to the sensitivity of PHQ- 9 and PHQ-2 in this context and age group. Still, even after controlling for depression, family violence robustly predicted suicidality. Suicide prevention programs should therefore not only focus on strategies around depression, but also include interventions engaging the home, school and community ${ }^{3}$ to strengthen self-efficacy, and increase access to social networks and institutional support systems (eg, helplines). ${ }^{36}$ Our findings also suggest that government and non-governmental entities should work at decreasing domestic violence and encouraging supportive parent-child dynamics as a means to improve adolescent mental health.

Future studies on adolescents in India should continue to focus on suicidality given the high burden and inadequate understanding of its determinants in LMICs. Data from the National Crime Records Bureau (NCRB) and the Sample Registration System (SRS) from the Indian Census show suicide as a leading cause of mortality among youth, likely to surpass maternal mortality as the leading cause of death among young women in coming years. ${ }^{37}$ Despite these staggering numbers, suicide mortality may be underestimated given reporting inconsistencies; the Mental Health Care Act in 2017 decriminalized suicide, ${ }^{38}$ which is a first step in improving the accuracy of reporting rates and reducing the stigma around reporting suicide. ${ }^{39}$ For this reason, suicidality studies based on population-level surveys such as this study can be instrumental in understanding the true burden and risk factors for suicidal ideation, self-harm, and depression among adolescents. ${ }^{37}$
Findings of this study should be interpreted keeping in mind three limitations. First, we conducted stratified analyses to account for developmental and gender differences among adolescents. Though appropriate and recommended due to the sampling design and survey weights, this practice led to smaller sample sizes (eg, $n=1,653$ for $10-14$ year old girls) which prohibited us from estimating interactions between family violence and social inequity markers like caste, religion and wealth. Second, adolescent reporting on violence was retrospective and non-specific. This meant our understanding of when an event occurred or the frequency that is occurred was limited and subject to recall bias. We focused on physical violence here but future studies should consider investigating associations with other forms of violence. Finally, we used PHQ-2 and PHQ-9 depression scores in relation to suicidality due to the cross-sectional nature of our data. This is less than ideal as the recall period for these instruments for measuring depression is the last 15 days and 12 months for suicidal ideation; the use of longitudinal data when available is preferred.

\section{CONCLUSIONS}

Adolescents in Bihar and Uttar Pradesh (UP), India report notable prevalence of suicidality, self-harm and depressive symptoms, which were more common among adolescent girls than boys, with exceptionally high reporting among married adolescent girls. Married adolescent girls (15-19 years) were most vulnerable in this context, likely to face both parental and spousal violence while younger boys ${ }^{10-13,20}$ were most likely to experience physical abuse. India's flagship adolescent health program RKSK needs to consider gender and developmentally sensitive interventions for preventing adolescent suicidality, self-harm and depression employing insights from a broader ecological perspective such that it includes family involvement and domestic violence prevention. Given the sheer size of India's adolescent population - $20 \%$ or 250 million people ${ }^{5}$ addressing adolescent mental health concerns could have a population-level impact on health in India.

\section{ACKNOWLEDGEMENTS}

Authors wish to thank Population Council and Harvard Dataverse for assistance with data access.

\section{ETHICS APPROVAL}

Information on ethics approval is included in the manuscript.

\section{FUNDING}

This work was supported by the David and Lucile Packard Foundation (Grant number: 2017-66705). 


\section{COMPETING INTERESTS}

The authors completed the Unified Competing Interest form at http://www.icmje.org/coi_disclosure.pdf (available upon request from the corresponding author), and declare no conflicts of interest.

\section{CORRESPONDENCE TO:}

Nandita Bhan

Research Scientist

Center on Gender Equity and Health 9500 Gilman Drive La Jolla,CA 92093

USA

nabhan@ucsd.edu 


\section{REFERENCES}

1. World Health Organization. Causes of Death among Adolescents (Data from WHO Global Health Estimates 2016. Published 2018. Accessed April 1, 2019. http://www.whoint/maternal_child_adolescent/ data/causes-death-adolescents/en/

2. Hawton K, Saunders KE, O’Connor RC. Self-harm and suicide in adolescents. Lancet. 2012;379:2373-282. doi:10.1016/S0140-6736(12)6032 $\underline{2-5}$

3. Patton GC, Sawyer SM, Santelli JS, Ross DA, Afifi R, Allen NB. Our future: a Lancet commission on adolescent health and wellbeing. Lancet. 2016;387:2423-278. doi:10.1016/S0140-6736(16)0057 9-1

4. Borges G, Nock MK, Abad JM, Hwang I, Sampson NA, Alonso J. Twelve-month prevalence of and risk factors for suicide attempts in the World Health Organization World Mental Health Surveys. J Clin Psychiatry. 2010;71:1617-128. doi:10.4088/JCP.08m04 967blu

5. G.O.I. Census of India. SRS Statistical Report 2015. Detailed Tables. Accessed April 1, 2019. http://censusi ndia.gov.in/DigitalLibrary/Tables.aspx

6. Dandona R, Kumar GA, Dhaliwal R, Naghavi M, Vos T. Shukla DK Gender differentials and state variations in suicide deaths in India: the Global Burden of Disease Study 1990-2016. Lancet Public Health. Published online 2018. doi:10.1016/S2468-2667(18)30 $\underline{138-5}$

7. Fazel M, Patel V, Thomas S, Tol W. Mental health interventions in schools in low-income and middleincome countries. Lancet Psychiatry. 2014;1:388-398. doi:10.1016/S2215-0366(14)70357-8

8. Barry MM, Clarke AM, Jenkins R, Patel V. A systematic review of the effectiveness of mental health promotion interventions for young people in low and middle income countries. BMC Public Health. 2013;13(835). doi:10.1186/1471-2458-13-835

9. World Health Organization. Preventing Suicide: A Global Imperative. World Health Organization; 2014.

10. Government of India. Rashtriya Kishor Swasthya Karyakram (RKSK). National Health Mission Ministry of Health \& Family Welfare, Government of India. Published 2014. Accessed April 1, 2019. http://nhm.g ov.in/rashtriya-kishor-swasthya-karyakram.html
11. Government of India. National Health Mission Components. Adolescent Heath (RKSK). Background. National Health Mission Ministry of Health \& Family Welfare, Government of India 2014. Published online 2014. Accessed April 1, 2019. http://www.nhm.gov.in/ nrhm-components/rmnch-a/adolescent-health-rksk/r ksk-background.html

12. Government of India. Rashtriya Kishor Swasthya Karyakram - Operational Framework. Published 2014. Accessed April 1, 2019. https://www.nhmgovin/index 1 php?lang $=1$ \&level $=4$ \& sublinkid $=293 \&$ lid $=153$

13. Roh BR, Jung EH, Hong HJ. A Comparative study of suicide rates among 10-19-year-olds in 29 OECD countries. P Psychiatry Investig. 2018;15(376). doi:10.3 $\underline{0773 / \text { pi.2017.08.02 }}$

14. Aggarwal S, Berk M. Evolution of adolescent mental health in a rapidly changing socioeconomic environment: a review of mental health studies in adolescents in India over last 10 years. Asian J Psychiatr. 2015;13:3-12. doi:10.1016/j.ajp.2014.11.007

15. Aggarwal S. Suicide in India. Br Med Bull. 2015;114:127-134. doi:10.1093/bmb/ldv018

16. Singhi P, Saini AG, Malhi P. Child maltreatment in India. Paediatr Int Child Health. 2013;33:292-300. do i:10.1179/2046905513Y.0000000099

17. IIPS. National Family Health Survey (NFHS-4), 2015-16. International Institute for Population Sciences (IIPS) and ICF; 2018.

18. Kidman R. Child marriage and intimate partner violence: a comparative study of 34 countries. Int J Epidemiol. 2016;46:662-675. doi:10.1093/ije/dyw225

19. Devries KM, Mak JY, Bacchus LJ, et al. Intimate partner violence and incident depressive symptoms and suicide attempts: a systematic review of longitudinal studies. PLoS Med. 2013;10(5):e1001439. doi:10.1371/journal.pmed.1001439

20. Government of India. Rashtriya Kishor Swasthya Karyakram (RKSK). Accessed April 1, 2019. https://ww w.nhmgovin/index1php?lang=1 \&level=4\&sublinki $\underline{d=293 \& l i d=153}$

21. MAMTA. Adolescent Health and Implementation Science: Achievements, Challenges and Prospects. New Delhi, India; 2016. 
22. Santhya KG, Acharya R, Pandey N, et al. Understanding the Lives of Adolescents and Young Adults (UDAYA) in Bihar, India. Population Council; 2017. doi:10.31899/pgy8.1045

23. Santhya KG, Acharya R, Pandey N, et al. Understanding the Lives of Adolescents and Young Adults (UDAYA) in Bihar, India. Population Council; 2017. doi:10.31899/pgy8.1045

24. Kroenke K, Spitzer RL. The PHQ-9: a new depression diagnostic and severity measure. Psychiatr Ann. 2002;32:509-515. doi:10.3928/0048-5713-20020 901-06

25. Kroenke K, Spitzer RL, Williams JB. The Patient Health Questionnaire-2: validity of a two-item depression screener. Med Care. 2003;41:1284-192. do i:10.1097/01.MLR.0000093487.78664.3C

26. Petroni S, Patel V, Patton G. Why is suicide the leading killer of older adolescent girls? Lancet. 2015;386:2031-22. doi:10.1016/S0140-6736(15)0101 9-3

27. Naved RT, Akhtar N. Spousal violence against women and suicidal ideation in Bangladesh. Womens Health Issues. 2008;18:442-452. doi:10.1016/j.whi.200 $\underline{8.07 .003}$

28. Raj A. When the mother is a child: the impact of child marriage on the health and human rights of girls. Arch Dis Child. 2010;95:931-995. doi:10.1136/ad c.2009.178707

29. Basu S, Zuo X, Lou C, Acharya R, Lundgren R. Learning to be gendered: gender socialization in early adolescence among urban poor in Delhi. India, and Shanghai, China J Adolesc Health.

2017;61:S24-9:24-29. doi:10.1016/i.jadohealth.2017.0 $\underline{3.012}$

30. Blum RW, Mmari K, Moreau C. It begins at 10: How gender expectations shape early adolescence around the world. J Adolesc Health. 2017;61:S3-4. do i:10.1016/i.jadohealth.2017.07.009
31. Runyan DK, Shankar V, Hassan F, et al. International variations in harsh child discipline. Pediatrics. 2010;126(3):e701-e711. doi:10.1542/peds.2 008-2374

32. Salk RH, Hyde JS, Abramson LY. Gender differences in depression in representative national samples: Meta-analyses of diagnoses and symptoms. Psychol Bull. 2017;143(783). doi:10.1037/bul0000102

33. Breslau J, Gilman SE, Stein BD, Ruder T, Gmelin T, Miller E. Sex differences in recent first-onset depression in an epidemiological sample of adolescents. Transl Psychiatry. 2017;7(5):e1139-e1139. doi:10.1038/tp.2017.105

34. Böge K, Zieger A, Mungee A, Tandon A, Fuchs LM. Schomerus $G$ Perceived stigmatization and discrimination of people with mental illness: A survey-based study of the general population in five metropolitan cities in India. Indian J Psychiatry. 2018;60(24). doi:10.4103/psychiatry.IndianJPsychiatr y_406_17

35. Lewinsohn PM, Rohde P, Seeley J. Psychosocial risk factors for future adolescent suicide attempts. $J$ Consult Clin Psychol. 1994;62(297). doi:10.1037/002 2-006X.62.2.297

36. Armstrong G, Vijayakumar L. Suicide in India: a complex public health tragedy in need of a plan. Lancet Public Health. 2018;3:e459-60:459-460. doi:1 $\underline{0.1016 / S 2468-2667(18) 30142-7}$

37. Patel V, Ramasundarahettige C, L. V, Thakur JS, Gajalakshmi V. Gururaj G Suicide mortality in India: a nationally representative survey. Lancet. 2012;379:2343-251. doi:10.1016/S0140-6736(12)6060 6-0

38. Government of India. The Mental Healthcare Act. The Gazette of India Ministry of Law and Justice, Government of India. Published online 2017. Accessed April 1, 2019. https://www.prsindia.org/recent-acts

39. Ranjan R, Kumar S, Pattanayak RD, Dhawan A, Sagar R. (De-) criminalization of attempted suicide in India: A review. I Ind Psychiatry J. 2014;23:4-9. doi:1 $\underline{0.4103 / 0972-6748.144936}$ 